\title{
MOON
}

\section{The mother of all bombardments}

Rocks gathered at the time of the Moon landings tell us that 2.9 Gyr ago our satellite sustained a period of intense impacts that formed its youngest basins like the large Mare Imbrium (pictured). Understanding the cause for this Late Heavy Bombardment (LHB) would give us important clues about the history of the Solar System. The community is divided between two main scenarios: the 'terminal cataclysm' exhibiting a surge of impacts at the time of the LHB, possibly generated by the migration of the giant planets; and the 'accretion tail', which would be the natural end of the decreasing trend of collisions among planetesimals since the formation of the Solar System. Numerical simulations, the lunar crater record and mineralogical evidence give contradictory indications of which scenario is the most likely. Alessandro Morbidelli and collaborators employ new results and up-to-date models to create a coherent history of the lunar bombardment (Icarus 305, 262-276; 2018).

One of the key elements of the study is the recent hypothesis that measurements of the lunar mantle abundance of highly siderophile elements (HSEs), one of the

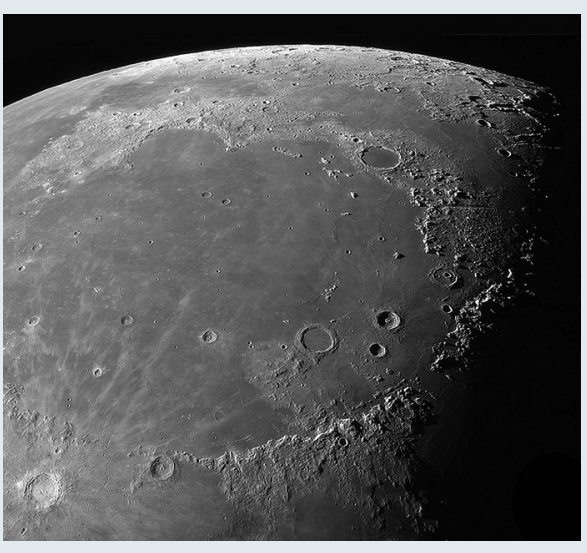

Credit: Joseph Brimacombe

main constraints for the lunar timeline, only account for the material accumulated in the mantle since the magma ocean crystallized. Setting the crystallization at a reasonable 4.4 Gyr ago (100-150 Myr after Moon formation), the model used by Morbidelli et al. shows that a LHB $\sim 3.95$ Gyr ago is fully consistent with the accretion tail scenario, without the need for a terminal cataclysm. This result fits nicely not only with the lunar constraints, but also with many of the various clues disseminated throughout the Solar System (the timing of the giant planets instability; the cratering history of Pluto and Charon as observed by New Horizons; the concentration of HSEs at Mars).

Of course, there are still some wrinkles. The timing does not seem to be compatible with the impact spike observed in the HED (howardite-eucrite-diogenite) meteorites, estimated at $4.1 \mathrm{Gyr}$ ago. It would also mean that the current crater record for the Moon and Mars significantly underestimates the actual number of impacts these two bodies received. The scenario proposed by Morbidelli et al. can, however, be confirmed - or not - by the precise determination of the age of the Martian northern basin (probably also produced by an impact early in the history of Mars) and of the lunar magma ocean crystallization. Both measurements should not be too far away in the future.

Luca Maltagliati

Published online: 27 February 2018 https://doi.org/10.1038/s41550-018-0421-4 